\title{
Aspectos perinatales de las adolescentes
}

\author{
EN LA CLINICA DE MATERNIDAD "RAFAEI_ CALVO C"(1) \\ Dres.: Jaime A. Barrios Amaya** Alvaro Ramos Olier** \\ Javier Arévalo Miranda*** Fernando Brown Morales*** \\ Alvaro Castro Castillo*** Raúl Herrera Chico*** \\ Oscar Fontalvo Malo*** Marcial Garzón Guzmán***
}

\section{INTRODUCCION}

La adolescencia es el período de transición entre la infancia y la edad adulta. Se caracteriza porque el individuo presenta un período de crecimiento físico rápido, busca liberarse de su familia, empieza a aceptar las responsabilidades propias y empieza a apartarse de las influencias de la casa; se interesa por su imagen corporal, aparecen nuevos impulsos sobre todo en el área sexual, y aparecen muchas inquietudes que intranquilizan a los jóvenes en esta edad (6).

Desde el punto de vista obstétrico, se consideran los problemas de las adolescentes como graves.

* Jefe, Departamento de Obstetricia y Gi. necología. Facultad de Medicina, Univer. sidad de Cartagena.

* Profesor Titular, Departamento de Obstetricia y Ginecología, Universidad de Cartagena.

** Estudiantes de 5o. año, Facultad de Medicina, Universidad de Cartagena.

(1). Este trabajo fue realizado en la Clínica de Maternidad "Rafael Calvo C", de la Universidad de Cartagena.

Presentado en el XV Congreso Colombiano de Obstetricia y Ginecología, Bucaramanga, diciembre de 1983.
Ciertos estudios mencionan que algunas complicaciones importantes durante el embarazo, como Toxemia, Anemia, Premadurez, Parto prolongado, y Complicaciones postparto, aumentan en esta etapa de la vida. Otros estudios más recientes demuestran que cuando se proporciona una amplia atención obstétrica disminuyen las complicaciones en este campo (7).

En América Latina $y$ en Colombia, particularmente, los estudios hechos sobre la Adolescencia son fragmentarios e incompletos, por ésto se hace necesaria una revisión y un estudio más a fondo de este problema en nuestro medio, el cual se puede hacer complementario y comparativo con otros trabajos investigativos sobre: Hemorragias de la 2a. mitad del embarazo, Recién nacidos de bajo peso, y Enfermedades hipertensivas durante la gestación.

Se considera que para una mayor atención obstétrica en la adolescente, es necesario un trabajo de equipo con la participación de padres, médico, enfermera, sicólogo, y trabajadora social. Además se deben iniciar campañas tendientes a impartir una adecuada educación sexual a temprana edad en nuestra población $(1,2,3,5)$. 
Las dos preocupaciones médicas que surgen por la conducta de los adolescentes son con mayor frecuencia el embarazo y las enfermedades venéreas.

En un estudio de mujeres de 15 a 19 años de edad, 9.3응 informaron de embarazo premarital. Más del 50 \% de estas mujeres llevan el embarazo a término, pero las cifras de quienes buscan aborto ha ido en aumento $(7,9)$.

¿Cómo solucionar estos problemas? Las posibilidades para actuar como consejero sexual son muy limitadas. Los adolescentes son renuentes a hablar de su vida sexual dentro de una relación convencional médico-paciente, pues considera al facultativo una prolongación de sus progenitores. Con empeño puede llegarse a muchos adolescentes y conocer sus preocupaciones, pero es menester conocer a fondo y precisar cuáles son estas preocupaciones a las que se enfrentan los adolescentes $(3,8)$.

\section{Embarazo y Aborto de la Adolescente. Factores Etiológicos}

Para los adolescentes de clase media, el embarazo es a menudo resultado de intentos frustrados por resolver los conflictos psicológicos específicos de su desarrollo. Cuando la patología psicológica o social grave es la causa del embarazo de la adolescente, el fenómeno se acompaña de otros patrones de maladaptación, como abuso de drogas, delincuencia, abandono de la familia o fuga de la escuela (6).

La ignorancia sobre las funciones se$x$ uales $y$ las supersticiones o temores relacionados con el uso de los anticonceptivos contribuyen también al problema del embarazo no deseado.

Una proporción pequeña de adolescentes desea el embarazo, y un número probablemente igual es indiferente a' la posibilidad del embarazo después del coito.

Para algunas de estas jóvenes el embarazo puede representar un intento constructivo de afrontar los problemas inherentes a la adolescencia. Por desgracia, es difícil que estas mujeres lleven a cabo más tarde un matrimonio estable, o que creen un ambiente saludable para el recién nacido, aunque algunas madres jóvenes logran triunfar. Los factores importantes en las que logran el triunfo son:

1. Existencia de una persona responsable que proporciona los medios de seguridad emocional y económica necesarios hasta que la adolescente se pueda bastar por sí misma.

2. Concepto realista del papel materno.

3. Motivación de que el embarazo ha siso por razones positivas y saludables y no por razones autodestructivas o neuróticas. Es útil valorar los factores personales, familiares y sociales que influyen en la adolescente embarazada, para que el médico se oriente hacia las alternativas disponibles para atender el embarazo y la posibilida'd de planear otros embarazos futuros.

Para la adolescente urbana el embarazo se complica además por pobreza, desajuste social y falta de comunicación. Los resultados por lo tanto dependen de que se conozcan los diversos factores etiológicos que intervienen, las necesidades específicas de la adolescente y la disponibilidad de diversos recursos comunitarios.

Medidas Sanitarias Preventivas: Las adolescentes embarazadas pueden ser consideradas como grupo de riesgo de repetición del embarazo, causando problemas sociales, educacionales y sanitarios futuros. Los niños producto de los 
embarazos de la adolescencia corren peligro de gran variedad de problemas, como abandono y desarrollo físico y emocional retrasado.

El éxito en la mayoría de las pacientes adolescentes depende en gran parte de los buenos servicios obstétricos, psicológicos, sociales y educacionales $(4,9)$.

La educación durante el embarazo, que comprende educación sexual, preparación emocional y actitud positiva hacia el embarazo, planeación familiar, nutrición, efectos de los fármacos en el embarazo, preparación para el parto planeación para el recién nacido, atención del lactante y vida familiar adecuada, puede ser muy valiosa para ayudar a la adolescente. Los programas educacionales que ofrecen asistencia psicológica pueden fomentar también una conducta más responsable y madura. Los contactos de la adolescente con el personal profesional durante el embarazo pueden ser su única fuente de información en cuanto a sexualidad, enfermedades venéreas y anticoncepción.

\section{MATERIAL Y METODOS}

Se revisaron 18.559 historias recopiladas en el lapso de 3 años comprendidos entre julio de 1980 y junio de 1983, en la Clínica de Maternidad "Rafael Calvo C". centro donde funciona el Departamento de Obstetricia y Ginecología de la Universidad de Cartagena.

De éstas, 2.070 historias correspondieron a pacientes menores de 18 años (parámetro de edad tomado para la adolescencia), las cuales consultaban por primera vez en este centro.

* La totalidad de los datos de las tablas fueron tomados de la Clínica de Maternidad "Rafael Calvo C" de la Universidad de Cartagena de julio de 1980 a junio de 1983.
Para el procesamiento de estos datos se utilizó la tabulación manual, y se tuvieron en cuenta los siguientes parámetros.

\section{Edad \\ Procedencia \\ Estavo civil \\ Historia Gineco-Obstétrica \\ Motivo de la Consulta \\ Diagnóstico de Egreso \\ Patologías Asociadas \\ Peso del Recién Nacido}

\section{RESULTADOS}

De las 18.559 pacientes admitidas por primera vez en la Clinica de Maternidad "Rafael Calvo" durante el período comprendido desde julio de 1980 a junio de 1983, sólo 2.070 pacientes eran adolescentes, es decir el 11.2\%. (Tabla No. 1).

Tabla No. 1

INCIDENCIAS DE

PACIENTES ADOLESCENTES

\begin{tabular}{|c|c|c|}
\hline >18 AÑOS & 16.489 & 888 \\
\hline ADOLESCENTES & 2.070 & 11.2 \\
\hline TOTAL & 18.559 & $100 \%$ \\
\hline
\end{tabular}

De los 2.070 casos estudiados, 1.938 fueron partos y abortos. El número de partos fue de 1.737 y el de abortos de 201 casos. El porcentaje de partos es de $89.6 \%$ y el de abortos de $10.4 \%$ ( Tabla No. 2).

Tabla No. 2

INCIDENCIA DE PARTOS Y ABORTOS EN PACIENTES ADOLESCENTES

\begin{tabular}{|c|c|c|}
\hline ENTIDAD & CASOS ESTUDIADOS & $\%$ \\
\hline PARTOS & 1.737 & 89.6 \\
\hline ABORTOS & 201 & 10.4 \\
\hline TOTAL & 1.938 & $100 \%$ \\
\hline
\end{tabular}


Durante los 3 años estudiados, hubo en la misma Clínica 33.234 casos entre partos y abortos, con una incidencia de partos de $87.7 \%$ ( 29.140 casos) y una incidencia de abortos de $12.3 \%$ (4.094 casos). En esta tabla no sólo se incluyen a las pacientes adolescentes sino también a las pacientes mayores de 18 años. (Tabla No. 3).

Tabla No. 3

TOTAL DE PARTOS Y ABORTOS

\begin{tabular}{|l|c|c|}
\hline ENTIDAD & $\begin{array}{c}\text { TOTAL CASOS } \\
\text { EN 3 ANNOS }\end{array}$ & $\%$ \\
\hline PARTOS & 29140 & 877 \\
\hline ABORTOS & 4.094 & 12.3 \\
\hline TOTAL & 23234 & $100 \%$ \\
\hline
\end{tabular}

De los 29.140 partos atendidos en la Clínica durante estos 3 años, 1.737 fueron de pacientes adolescentes, correspondiendo a un $5.96 \%$ del total de partos. El número de abortos fue de 4.094, de los cuales 201 casos en adolescentes, correspondiendo a un $4.9 \%$ del total de abortos. (Tabla No. 4).

Tabla No. 4

RELACION ENTRE

LAS TABLAS No. 2 Y No. 3

\begin{tabular}{|c|c|c|c|c|}
\hline$E A T D D A=$ & $\begin{array}{l}\text { TCTAL CDSCE } \\
\text { EN } 3 \text { ANNUS }\end{array}$ & $\begin{array}{l}\text { CASOS EN } \\
\text { ADOLESCENTES }\end{array}$ & & $\%$ \\
\hline PARTOS & 29140 & 1. $7 \geq 7$ & 5 & $9 \varepsilon$ \\
\hline ABORTOS & 4094 & 201 & 4 & 90 \\
\hline TOTAL & 33.234 & 1. 938 & 5 & 83 \\
\hline
\end{tabular}

El mayor número de pacientes estudiadas tenía 17 años, con 1.174 casos (56.71\%). Luego siguió el grupo de16 años, con 621 casos (30\%), el de 15 años con 213 casos $(10.29 \%)$, el de 14 años con 45 casos $(2.17 \%$ ), el de 13 años con 13 casos $(0.63 \%)$ y el de 12 y 11 años con 2 casos (0.1\%). (Tabla No. 5).
Tabla No. 5

DISTRIBUCION POR EDAD

DE PACIENTES ADOLESCENTES

\begin{tabular}{|c|c|c|}
\hline EDAD & No & $\%$ \\
\hline 11 & 2 & 0.1 \\
\hline 12 & 2 & 0.1 \\
\hline 13 & 45 & 063 \\
\hline 14 & 213 & 217 \\
\hline 15 & 621 & 1029 \\
\hline 16 & 1174 & 300 \\
\hline 17 & 2.070 & 56.71 \\
\hline TOTAL & & $100 \%$ \\
\hline
\end{tabular}

La mayoría de las pacientes fueron de procedencia urbana, con $73.6 \%$ y las pacientes de procedencia rural fueron el $22.4 \%$. En 32 historias $(4 \%)$ no se registró este dato. (Tabla No. 6).

Tabla No. 6

PROCEDENCIA

DE PACIENTES ADOLESCENTES

\begin{tabular}{|c|c|c|}
\hline URBANA & $15 E 4$ & $73.0 \%$ \\
\hline RUAAL & 4.4 & $22.4 \%$ \\
\hline S DATOS & 82 & $4 \%$ \\
\hline & 2.070 & $100 \%$ \\
\hline
\end{tabular}

Las adolescentes urbanas se distribuyeron principalmente en los sectores marginados de la ciudad, como son la zona sur oriental, con un $64.9 \%$. El resto residian en los barrios cercanos a la Clínica de Maternidad (19.85\%) y en el sur de la ciudad $(11.89 \%)$. Pocos casos corresponden a los barrios de clase media-alta y alta (3.36\%). (Figura No. 1).

La mayoría de las pacientes tuvieron su menarquia entre los 12 y 14 años de 
edad, con mayor incidencia a los 13 años, 619 casos (29.9\%), luego a los 12 años, 517 casos (25\%), a los 14 años, 414 casos (20\%), a los 11 años, 180 casos (8.7\%), a los 17 años, 144 casos (6.95\%), a los 15 años, 122 casos (2.02응, a los 16 años, 18 casos $(0.86 \%)$, a los 9 años, 13 casos $(0.62 \%)$ y sólo un caso tuvo menarquia a los 7 años $(0.05 \%)$.La edad promedio fue de 12.4 años. (Tabla No. 7).

El $70 \%$ de los casos (1.448) eran madres solteras, y el $23.3 \%$ eran madres casadas (462 casos). Hubo 160 historias $(6.7 \circ)$ que no registraron este dato. (Tabla No. 8).

La edad en que con mayor frecuencia se inició la vida marital fue a los 16 años, con 618 casos (35.72\%). Sigue el grupo de 15 años, con 431 casos (24.9\%), luego el de 17 años con 278 casos (16.06\%), el de 14 años con 165 casos (9.55\%), el de 13 años con 38 casos (2.2\%) el de 12 años con 16 casos (0.92\%), y en 4 casos se inició vida marital a los 11 años. Hay 180 historias $\sin$ el registro

Figura No. 1

PLANO DE CARTAGENA DE INDIAS-DISTRIBUCION SECTORIA'L Y PORCENTUAL SEGUN PROCEDENCIA URBANA DE LAS ADOLESCENTES

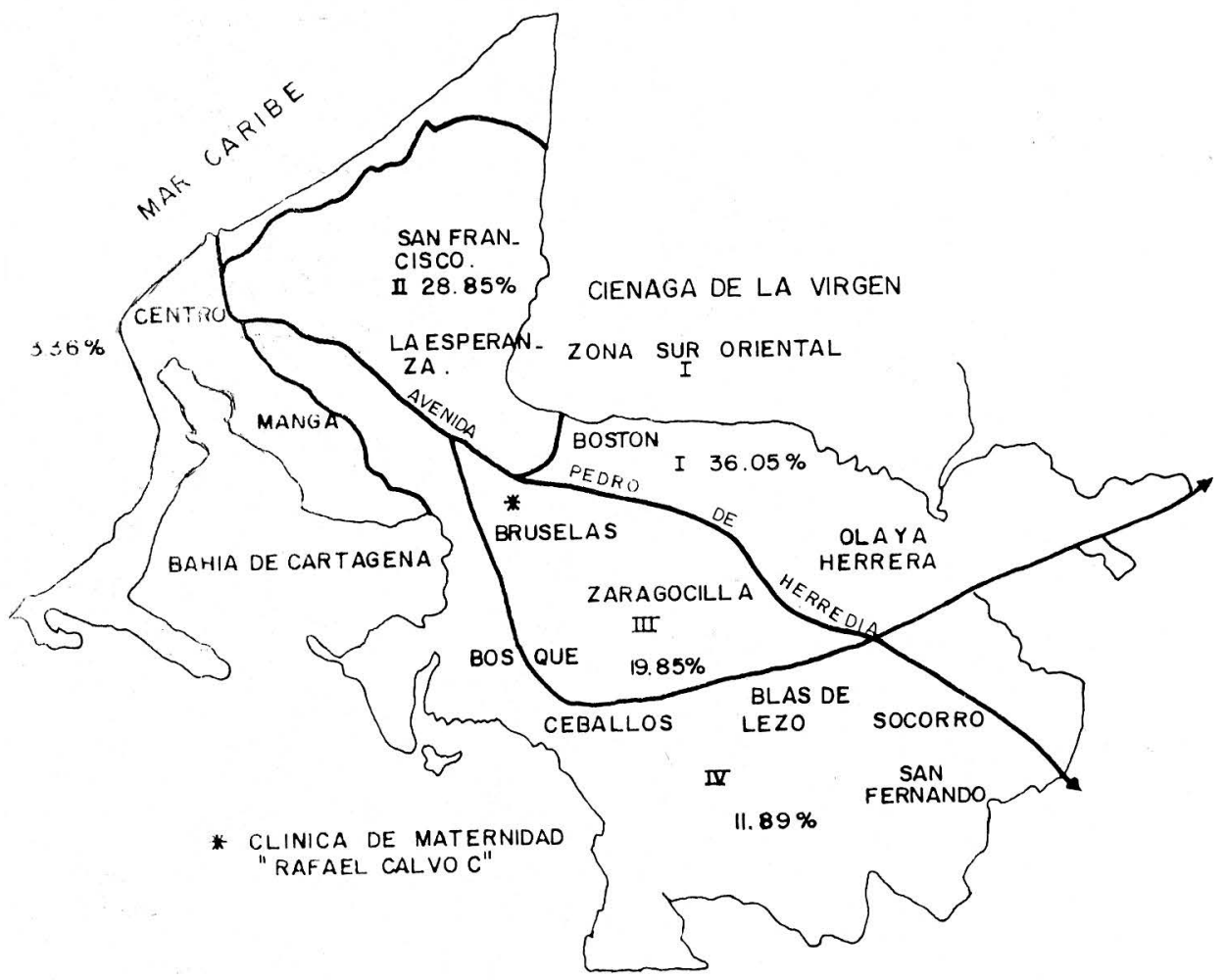


de inicio de vida marital, que corresponden al $10.7 \%$ (Tabla No. 9).

\section{Tabla No. 7}

MENARQUIA SEGUN

LA EDAD EN ADOLESCENTES

\begin{tabular}{|c|c|c|}
\hline$E D A C$ & CASOS & $\%$ \\
\hline 7 & 1 & 0.05 \\
\hline 3 & 13 & 062 \\
\hline 10 & 42 & 2.02 \\
\hline 11 & 180 & 8.70 \\
\hline 12 & 517 & 25.00 \\
\hline 13 & 619 & 29.90 \\
\hline 14 & 414 & $20 \mathrm{C}$ \\
\hline 15 & 122 & 5.9 \\
\hline 16 & 18 & 0.86 \\
\hline 17 & 144 & 6.95 \\
\hline TOTAL & 2.070 & $100 \%$ \\
\hline
\end{tabular}

Tabla No. 8

ESTADO CIVIL EN ADOLESCENTES

\begin{tabular}{|c|c|c|}
\hline $\begin{array}{c}\text { ESTADO } \\
\text { CIVIL }\end{array}$ & CASOS & $\%$ \\
\hline SOLTERAS & 1448 & 70 \\
\hline CASADAS & 462 & 23.3 \\
\hline S. DATOS & 160 & 6.7 \\
\hline TOTAL & 2070 & $100 \%$ \\
\hline
\end{tabular}

La vida obstétrica se inició más frecuentemente a los 17 años, 672 casos (38.95\%). Sigue después el grupo de 16 años, 520 casos $(30 \%)$, luego los 15 años, 275 casos (15.9\%), los 14 años, 73 casos (4.2\%), los 13 años, 21 casos (1.2\%), los 12 años, 1 caso $(0.052 \%), y$ 2 casos a los 11 años $(0.1 \%)$. Hubo 166 casos (9.6\%) sin este registro. (Tabla No. 10).
Tabla No. 9

VIDA MARITAL EN ADOLESCENTES

\begin{tabular}{|c|c|c|}
\hline EDA & CASCS & $\%$ \\
\hline$\therefore$ & 4 & 025 \\
\hline 12 & $1 E$ & 0.92 \\
\hline 13 & 38 & 2.20 \\
\hline 14 & 165 & 9.55 \\
\hline 15 & 431 & 24.90 \\
\hline $1 E$ & 618 & 35.72 \\
\hline 17 & 278 & 1606 \\
\hline SE & $18 C$ & 10.40 \\
\hline TO TAL & 1.730 & $100 \%$ \\
\hline
\end{tabular}

Tabla No. 10

VIDA OBSTETRICA EN ADOLESCENTES

\begin{tabular}{|c|c|c|}
\hline EDAD & CASOS & $\%$ \\
\hline 11 & 2 & 0.10 \\
\hline 12 & 1 & 0.05 \\
\hline 13 & 21 & 1.20 \\
\hline 14 & 275 & 4.20 \\
\hline 15 & 520 & 15.90 \\
\hline 16 & 672 & 3000 \\
\hline 17 & 160 & 36.95 \\
\hline S.D & 1730 & 940 \\
\hline $10 \mathrm{TAL}$ & $90 \%$ \\
\hline
\end{tabular}

De las 2.070 pacientes, llegaron 1.874 primigrávidas, o sea $90.5 \%$ de los casos; 134 fueron secundigestantes $(6.5 \%), 58$ pacientes habían estado embarazadas 4 veces $(2.8 \%)$, y 4 habían gestado 3 veces (0.2\%). (Tabla No. 11).

Del total de partos, 1.745 pacientes tuvieron su primer parto $(92.4 \%) ; 24$ 
pacientes tuvieron su segundo parto (4.4\%) y sólo 2 pacientes tuvieron su tercer parto $(0.1 \%)$. Hubo 58 casos que no registraron el dato de paridad (3.1\%). (Tabla No. 12).

Tabla No. 11

GRAVIDEZ EN ADOLESCENTES

\begin{tabular}{|c|c|c|}
\hline GRAVIDEZ & CASOS & $\%$ \\
\hline$G$ & 1874 & 90.5 \\
\hline 6 & 134 & 6.5 \\
\hline G:3 & 4 & 0.2 \\
\hline TOTAL & 2.070 & 2.8 \\
\hline
\end{tabular}

Del total de pacientes, hubo 201 casos de aborto, de los cuales 192 eran el primer aborto (74.1\%), y 9 casos tenían historia de aborto anterior (3.5\%). Hubo 58 casos (22.4\%) donde no se registró este dato. (Tabla No. 13).

Tabla No. 12

PARIDAD EN ADOLESCENTES

\begin{tabular}{|c|c|c|}
\hline PARIDAD & CASOS & $\%$ \\
\hline$P 1$ & 1.745 & 924 \\
\hline 2 & 84 & 44 \\
\hline 3 & 2 & 01 \\
\hline$S D$ & 58 & 3.1 \\
\hline TOTAL & 1.889 & $100 \%$ \\
\hline
\end{tabular}

El más frecuente motivo de consulta fue el dolor de parto, 1.767 pacientes $(85.36 \%)$, Siguió luego el sangrado por genitales externos, 229 casos (11.06\%), la retención placentaria, 10 casos $(0.48 \%)$, convulsiones, 12 casos $(0.58 \%)$, y otros, que formaron 52 casos $(2.52 \%)$. Tabla No. 14).
Tabla No. 13

ABORTOS EN ADOLESCENTES

\begin{tabular}{|c|c|c|}
\hline ABERTOS & CASOS & $\%$ \\
\hline$A 1$ & 192 & 741 \\
\hline$A 2$ & 8 & 35 \\
\hline$S 0$ & 58 & 224 \\
\hline$T O R \therefore L$ & 259 & $100 \%$ \\
\hline
\end{tabular}

Tabla No. 14

MOTIVO DE CONSULTA

EN ADOLESCENTES

\begin{tabular}{|c|c|c|}
\hline$M O T V O E$ & CASCS & $\%$ \\
\hline DOLCPES DE PARTE & 767 & 8536 \\
\hline SANGRADO POR GENITALES EXT. & 220 & 11.06 \\
\hline RETENCION PLACENTARIA & 10 & 0.48 \\
\hline CONVULSIONES & 12 & 0.58 \\
\hline OTROS & 52 & 2.52 \\
\hline TOTA L & 2.070 & $100 \%$ \\
\hline
\end{tabular}

El parto vaginal normal fue el más frecuente, 1.552 casos (89.35\%), luego hubo 107 casos de cesárea (6.15\%), 49 partos conducidos $(2.8 \%), 19$ partos intervenidos $(1.1 \%$ y 5 casos inducidos $(0.3 \%)$. Hubo 5 casos en que el parto fue conducido e intervenido (0.3\%). (Tabla No. 15).

Tabla No. 15

DIFERENTES FORMAS

DE PARTO EN ADOLESCENTES

\begin{tabular}{|c|c|c|}
\hline$T \perp P O S$ & CASOS & $\%$ \\
\hline FAFTC AAUMAL NCRNAL & $: 5 E \hat{\imath}$ & 8935 \\
\hline 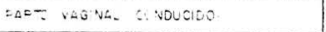 & 45 & 28 \\
\hline FARTO UAGINA NOJCIDO & 5 & 0.3 \\
\hline FARTS VAG:NAL INTERVENIDO & $: 9$ & 1. 1 \\
\hline PARTO VAGINA CONDUCIDO MAS NTERVINIDO & 5 & 0.3 \\
\hline CESAREAS & 107 & 6.15 \\
\hline $\begin{array}{lllll}T & 0 & T & A & L\end{array}$ & 1737 & $100 \%$ \\
\hline
\end{tabular}


La mayoría de los recién nacidos tuvieron un peso entre 2.500 y 3.500 gramos, con 1.198 casos $(68.77 \%$ ). Hubo 179 recién nacidos de bajo peso (10.27\%), y 271 casos de peso mayor de 3.500 gramos (15.55\%). No se registró el peso de 94 recién nacidos (5.41\%).

\section{Tabla No. 16}

PESO DE RECIEN NACIDOS

EN ADOLESCENTES

\begin{tabular}{|l|c|c|}
\hline FESO EN GR & CA SOS & $i_{c \mid}$ \\
\hline$<2500$ & 70 & 1027 \\
\hline 250003.500 & 1198 & 6877 \\
\hline$>3.500$ & 271 & 15.55 \\
\hline SIN DATOS & 94 & 5.41 \\
\hline TO TA L & 1.742 & $100 \%$ \\
\hline EMBARAZOS & GEMELARES & $5-$ \\
\hline
\end{tabular}

Tabla No. 17

COMPLICACIONES PRESENTADAS EN ADOLESCENTES

\begin{tabular}{|c|c|c|}
\hline COMPLICACIONES & No & $\%$ \\
\hline ENFERMED HIPERTENSIVA DEL EMBARAZO & 145 & 257 \\
\hline FUFT:FL PFEMATURA DE MEMBRANA & $: 7$ & 207 \\
\hline MIPOOINAMIAS & 59 & 105 \\
\hline PARTOS PREMATUROS & 52 & 9.2 \\
\hline OESPROPORCION CEFALC PELVICA IOC.PI) & 50 & 8.9 \\
\hline PRESENTACIONES ANOMALA & $4 i$ & 7.2 \\
\hline LMNIDNTIS & 3 & 5.5 \\
\hline RETENIION OE RESTOS PLACENTIRIOS & is & $2 E$ \\
\hline ENDOMETRTIS PUERPERAL & $\cdot E$ & 28 \\
\hline INFECC ON LIFINAEL $L$ & $\therefore$ & 25 \\
\hline$n=p+1$ & $\vdots$ & $\therefore$ \\
\hline 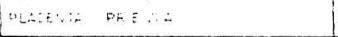 & : & $\therefore$ \\
\hline 1/OLA & 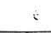 & 10 \\
\hline DTROS & 5 & 0.9 \\
\hline$T O T A L$ & 564 & $100 \%$ \\
\hline
\end{tabular}

Se presentaron complicaciones obstétricas en 564 de las 2.070 pacientes estudiadas $(27.2 \%)$. La complicación más frecuente fue la enfermedad hipertensiva del embarazo, con 145 casos $(25.7 \%$ de los 564), siguió la ruptura prematura de membranas con 117 casos (20.7\%), 59 casos de hipodinamia (10.5\%), 52 casos de partos prematuros $(9.2 \%), 50$ casos de desproporción céfalo-pélvica (8.9\%), 41 casos de presentaciones anómalas $(7.2 \%), 31$ casos de amnionitis (5.5\%), 16 de retención placentaria (2.8\%), 16 de endometritis puerperal $(2.8 \%$ y 14 casos de infección urinaria (2.5\%). De desprendimiento prematuro de placenta normoinserta, placenta previa y mola hidatiforme, hubo 6 casos en cada entidad ( $1 \%$ para cada patología). Hubo 5 casos con otras complicaciones, que correspondieron al $0.9 \%$ de los 564 casos con patologías obstétricas. (Tabla No. 17).

\section{DISCUSION}

Se revisaron en nuestro estudio 18.599 historias de pacientes admitidas en la Clínica de Maternidad "Rafael Calvo C" por primera vez, durante un lapso de 3 años. Del total, 2.070 historias correspondieron a pacientes adolescentes, que representan el $11.2 \%$ del total.

De las pacientes adolescentes estudiadas se encontró que el $73.6 \%$ procedía del área urbana, y el 22.4 del área rural.

De ésto se deduce que la cobertura de la atención obstétrica de la Clínica de Maternidad "Rafael Calvo $C$ " no se circunscribe a los límites urbanos de la ciudad de Cartagena, sino que se extiende al área rural, en un porcentaje significativo, ya que constituye la quinta parte de los casos estudiados.

Encontramos que la edad promedio de menarquia en nuestro estudio fue de 12.4 años, y que el $90 \%$ de las pacientes menstruó por vez primera entre los $11 \mathrm{y}$ 
los 15 años, con edades límites de 7 a 17 años. Estos resultados coinciden con los encontrados por Ucrós Cuellar (8).

En nuestro estudio hallamos que el $70 \%$ de las pacientes eran solteras y el 23응 eran casadas, con 17 o menos años.

La edad de inicio a la vida marital de las pacientes estudiadas fue de 14 años en promedio, con un límite inferior de 11 años y una mayor incidencia a los 16 años. Esto demuestra la iniciación sexual precoz de la adolescente en nuestro medio.

El inicio de la vida obstétrica tuvo una edad promedio de 16 años, con una mayor incidencia a los 17 años, y un límite inferior de 11 años, estando el 85\% de las pacientes entre los 15 y los 17 años. El $90.5 \%$ de las pacientes eran primigestantes. Estos datos son importantes ya que la gestación en esta etapa de la vida es considerada de alto riesgo, lo cual es puesto de manifiesto por la frecuencia de las complicaciones materno fetales encontradas en nuestra casuística (27.2\%) que fueron así: Enfermedad hipertensiva del embarazo, 25.7\% de las pacientes complicadas; Ruptura prematura de membranas, 20.7\%; Hipodinamias, 10.5\%; Partos prematuros 9.2\%, Desproporción céfalo-pélvica $8.9 \%$ y otras como Presentación anómala, Amnionitis; Retención de restos placentarios, Endometritis puerperal, Infección urinaria, D.P.P.N.I., P.P. y mola, que corresponden al $24.8 \%$ del total de complicaciones. Concluimos que la gestación en la adolescente con una adecuada atención no debe ser motivo de incremento de la morbilidad materno-fetal.

De los partos estudiados, sólo el $6.15 \%$ fue de tipo abdominal, y el $89.35 \%$ de tipo vaginal normal. Deducimos así que la adolescente puede tener un parto vaginal nurmal, aunque se considere que ella no tiene un grado de maduración pélvica óptimo para parir.
En cuanto al peso del neonato, encontramos que la mayoría (68.77\%) correspondieron a la escala comprendida entre 2.500-3.500 gramos; los que pesaron más de 3.500 gramos (15.55\%) superaron a los recién nacidos de bajo peso (menos de 2.500 gramos), los cuales representaron sólo el $10.27 \%$ del total.

\section{CONCLUSIONES Y RECOMENDACIONES}

De la presente revisión realizada en la Clínica de Maternidad "Rafael Calvo C", se concluye en forma definitiva que es importante realizar un diagnóstico $y$ atención temprana para las adolescentes con problemas obstétricos, si tenemos en cuenta que estas pacientes son consideradas de alto riesgo.

Se deben iniciar campañas tendientes a llevar una educación sanitaria en las áreas de sexualidad, reproducción y anticoncepción, ya que observamos que son gentes de escasos recursos económicos, con poca educación al respecto, y que consideran el tema sexual como tabú.

Se debe preparar a las adolescentes para actividades maternales $y$ de buena atención al recién nacido.

Debemos mejorar la vigilancia en los servicios de planificación, en las entidades nombradas por el gobierno para tal fin.

Hay que recalcar la importancia de la visita de la mujer embarazada a los centros de atención adecuados para ello, con la seguridad de que se disminuirán las complicaciones pre y post parto.

La edad promedio de aparición de la menarquia en nuestro medio tiene pocas variaciones comparada con otras ciudades del país que poseen semejanzas ecológicas, antropológicas y culturales. 
Se demuestra la importancia de la Clínica de Maternidad "Rafael Calvo C" como centro de atención obstétrico que cobija a toda nuestra provincia. Se debe mejorar la vigilancia obstétrica en el área rural.

No se puede concluir si la mayoría de los abortos en las adolescentes son provocados, ya que estos datos no se consignan en las historias elaboradas en la Clínica.

El promedio disminuido de recién nacidos de bajo peso en pacientes adolescentes, nos permite concluir que no es importante la relación de recién na- cidos de bajo peso con la edad de la madre.

Recomendamos a los directivos de la institución la incorporación en la historia clínica de ciertos datos como:

Escolaridad de la paciente, determinar en casos de aborto si éste es provocado y la causa, recalcar al personal encargado de llenar estas historias la importancia que tienen todos los datos para fines estadísticos.

Los problemas obstétricos en las adolescentes necesitan una atención integral para su solución.

\section{PERINATAL ASPECTS IN ADOLESCENT MOTHERS AT "RAFAEL CALVO" MATERNITY HOSPITAL}

\section{SUMMARY}

This article presents the conclusions obtained after a thorough assessment at "Rafael Calvo" Maternity Hospital, where it became evident that early diagnosis and treatment of adolescent women with obstetrical problems is of utmost importance, given the fact that they are considered high risk patients.

Since most of the patients are lowincome, they have little or no education on sexuality, reproduction and contraception, and consider the topic of sex a tabou. Health education campaigns focused on those areas is a priority.

Adolescents should also be prepared for maternal activities and actions and they must be capable of providing adequate attention to the newborn.

There should be greater effort in the appropriate institutions to supervise the activities and services provided by family planning agencies.
Antenatal care is considered a very important factor in reducing perinatal and post-natal complications.

The average age of menarche is similar in most colombian regions where ecological, anthropological and cultural traits are very much alike.

The article underlines the importance of "Rafael Calvo" Maternity Hospital in providing obstetric coverage in that region. Nevertheleses, it is important to improve coverage in the rural areas.

There ins't enough information to conclude if in the majority of cases abortion in adolescents are induced or not, since this piece of information is not recorded in the medical chart.

The average reduction of low birth weight suggests that there is no significant relationship between low birth weight and age of the mother. 
The authors highly recommend that the medical chart include more sociodemographic data, such as: level of formal education; in case of abortion, determine if it was induced, and what were the reasons that led to pregnancy termination; stress the importance of good

\section{BIBLIOGRAFIA}

1. AHUED-AHUED, J.R., LOWENBERG, E., VARGAS, C., TOMASI, E. Atención obstétrica en la adolescente. VIII Congr. Mundial Obst. Ginec. México. Octubre 1976.

2. AYLLON, R., ESCALANTE, J.M., GONZALEZ, J.M., DE LA LASTRA. La adolescencia y la edad avanzada como factores de riesgo en Obstetricia. VIII Congr. Mundial Obst. Ginec., México., Octubre 1976.

3. CORDOBA, R. Reflexiones acerca de la atención integral del adolescente. Antioquia Médica, 1974.

4. RIZO, A., PRADA, E. La fecundidad de la población de 10-19 años en Colombia, Rev. Col. Obstet. Ginec., Vol. XXXIII, No. 6, pp. 357-365, 1982. records for statistical and research purposes.

Obstetrical problems in adolescents require comprehensive care and an integral approach.

5. SANTIGAÑEZ, G., PEREZ, J.L., KUNHARDT, J., POHLS, V. Embarazo y parto en la adolescente. VIII Congr. Mundial Obst. Ginec., México, Octubre 1976.

6. Simposio sobre Medicina de Adolescentes Clin. Med. de Norteamérica, México., 1975, pp. 1283-1529.

7. Simposio sobre Medicina de Adolescentes Clin. Pediat. de Norteamérica, México, 1980, 1: 1-120.

8. UCROS CUELLAR, A., CASAS, L., UCROS, S. El adolescente en Colombia, Medicina, Bogotá, 5: 11-23, 1982.

9. VELASCO FERNANDEZ, R. Los problemas del adolescente. Salud Pública de México, 15: (2) 259-262, 1974. 